\title{
Improved VLBI astrometry of OH maser stars ${ }^{\star}$
}

\author{
W. H. T. Vlemmings ${ }^{1}$ and H. J. van Langevelde ${ }^{2,3}$ \\ 1 Argelander Institute for Astronomy, University of Bonn, Auf dem Hügel 71, 53121 Bonn, Germany \\ e-mail: wouter@astro.uni-bonn.de \\ 2 Joint Institute for VLBI in Europe, Postbus 2, 7990 AA Dwingeloo, The Netherlands \\ 3 Sterrewacht Leiden, Postbus 9513, 2300 RA Leiden, The Netherlands
}

Received 16 May 2007 / Accepted 4 July 2007

\begin{abstract}
Aims. Accurate distances to evolved stars with high mass loss rates are needed for studies of many of their fundamental properties. However, as these stars are heavily obscured and variable, optical and infrared astrometry is unable to provide enough accuracy. Methods. Astrometry using masers in the circumstellar envelopes can be used to overcome this problem. We have observed the $\mathrm{OH}$ masers of a number of Asymptotic Giant Branch (AGB) stars for approximately 1 year with the Very Long Baseline Array (VLBA). We have used the technique of phase referencing with in-beam calibrators to test the improvements this technique can provide to Very Long Baseline Interferometry (VLBI) OH maser astrometric observations.

Results. We have significantly improved the parallax and proper motion measurements of the Mira variable stars U Her, S CrB and RR Aql.

Conclusions. It is shown that both in-beam phase-referencing and a decrease in solar activity during the observations significantly improves the accuracy of the astrometric observations. The improved distances to S CrB $\left(418_{-18}^{+21} \mathrm{pc}\right)$ and RR Aql (633 $\left.3_{-128}^{+214} \mathrm{pc}\right)$ are fully consistent with published $P-L$ relations, but the distance to U Her $\left(266_{-28}^{+32} \mathrm{pc}\right)$ is significantly smaller. We conclude that for sources that are bright and have a nearby in-beam calibrator, VLBI OH maser astrometry can be used to determine distances to OH masing stars of up to $\sim 2 \mathrm{kpc}$.
\end{abstract}

Key words. masers - stars: circumstellar matter - stars: AGB and post-AGB - techniques: interferometric - astrometry

\section{Introduction}

VLBI astrometric observations of circumstellar OH masers can be used to determine the proper motion and parallax of enshrouded maser bearing stars (van Langevelde et al. 2000; Vlemmings et al. 2003, hereafter vL00 and V03). Obtaining accurate distances to these stars allows the inclusion of the more extreme Mira stars in studies of the fundamental properties of these stars, like the pulsation and mass-loss mechanism. Accurate parallaxes also allow the growing number of optical and infrared interferometric observations of AGB stars to be used to determine actual stellar sizes (e.g. Mennesson et al. 2002; Wittkowski et al. 2005). Currently, most investigations of pulsating stars are dependent on Hipparcos (Perryman et al. 1997), which is biased against the highly obscured stars with high mass loss. Unfortunately, as these stars are variable, the Hipparcos distances are often very uncertain. Maser astrometry can remedy this situation. In order to use the maser positions to monitor the stellar trajectory, an assumption has to be made about the motion of the masers with respect to the star. In V03, it was shown that for several stars the brightest, most blue-shifted circumstellar maser spot corresponds to the Amplified Stellar Image, as was previously hypothesized by Norris et al. (1984) and Sivagnanam et al. (1990). However, not all stars show such a maser spot, depending on for instance an inhomogeneous distribution of the masing gas (Vlemmings 2002). Still the observations in V03 indicate that even without an amplified stellar image, VLBI astrometry of $\mathrm{OH}$ masers yields highly accurate

* Figures 6-10 and Table 3 are only available in electronic form at http://www. aanda.org parallax and proper motion results. The only assumption needed for this is that these spots have linear motions in the shell.

The technique of VLBI astrometry has undergone extensive developments in the past several years. The feasibility of annual parallax measurements has now been proven at several different frequencies. At frequencies around $1.6 \mathrm{GHz}$, current astrometric observations provide pulsar velocities and distances out to several kpc (e.g. Brisken et al. 2002; Chatterjee et al. 2005). At higher frequencies, $\mathrm{H}_{2} \mathrm{O}$ and methanol maser astrometry is being used to obtain star-forming region distances up to several kpc with close to $\sim 2 \%$ accuracy (e.g. Hachisuka et al. 2006; Xu et al. 2006). $\mathrm{H}_{2} \mathrm{O}$ maser observations have also recently been used to measure the parallax of the Mira star UX Cyg (Kurayama et al. 2005) and additional $\mathrm{H}_{2} \mathrm{O}$ and $\mathrm{SiO}$ maser AGB astrometric observations are expected from the VERA project (Honma et al. 2003).

In this paper we present a follow-up on our earlier successful VLBI OH maser astrometry campaign (vL00, V03). In Sect. 2 we discuss the observations, data reduction and error analysis. In Sect. 3 we give the results of our parallax and proper motion determination. The results are compared with previous distance measurements in Sect. 4 along with a discussion about the internal maser motions in U Her. The conclusions are presented in Sect. 5.

\section{Observations and data reduction}

The positions of the $1.6 \mathrm{GHz}$ circumstellar $\mathrm{OH}$ masers of a sample of 6 AGB stars were monitored at 6 epochs spaced by 2-3 months between February 2004 and April 2005. The 

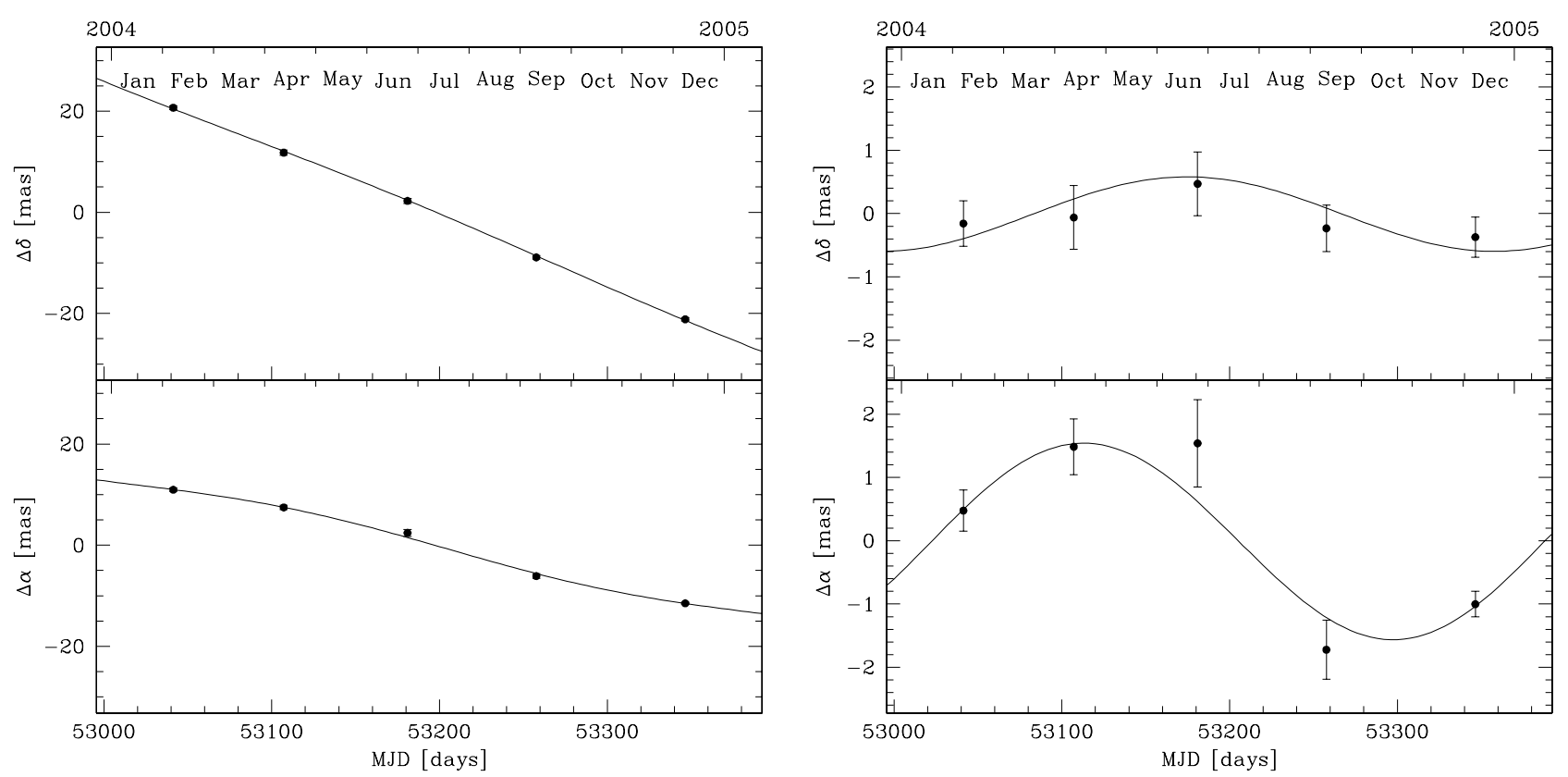

Fig. 1. The position of the brightest $1665 \mathrm{MHz}$ maser spot of RR Aql with respect to the in-beam calibrator (NVSS J195655-013615). The error bars on the positions indicate the position fitting errors described in Sect. 2.4. (Left) The solid line is the best fitting parallax and proper motion trajectory for the 5 epochs with usable observations. (Right) The best fitted parallax signature after subtracting the fitted proper motions.
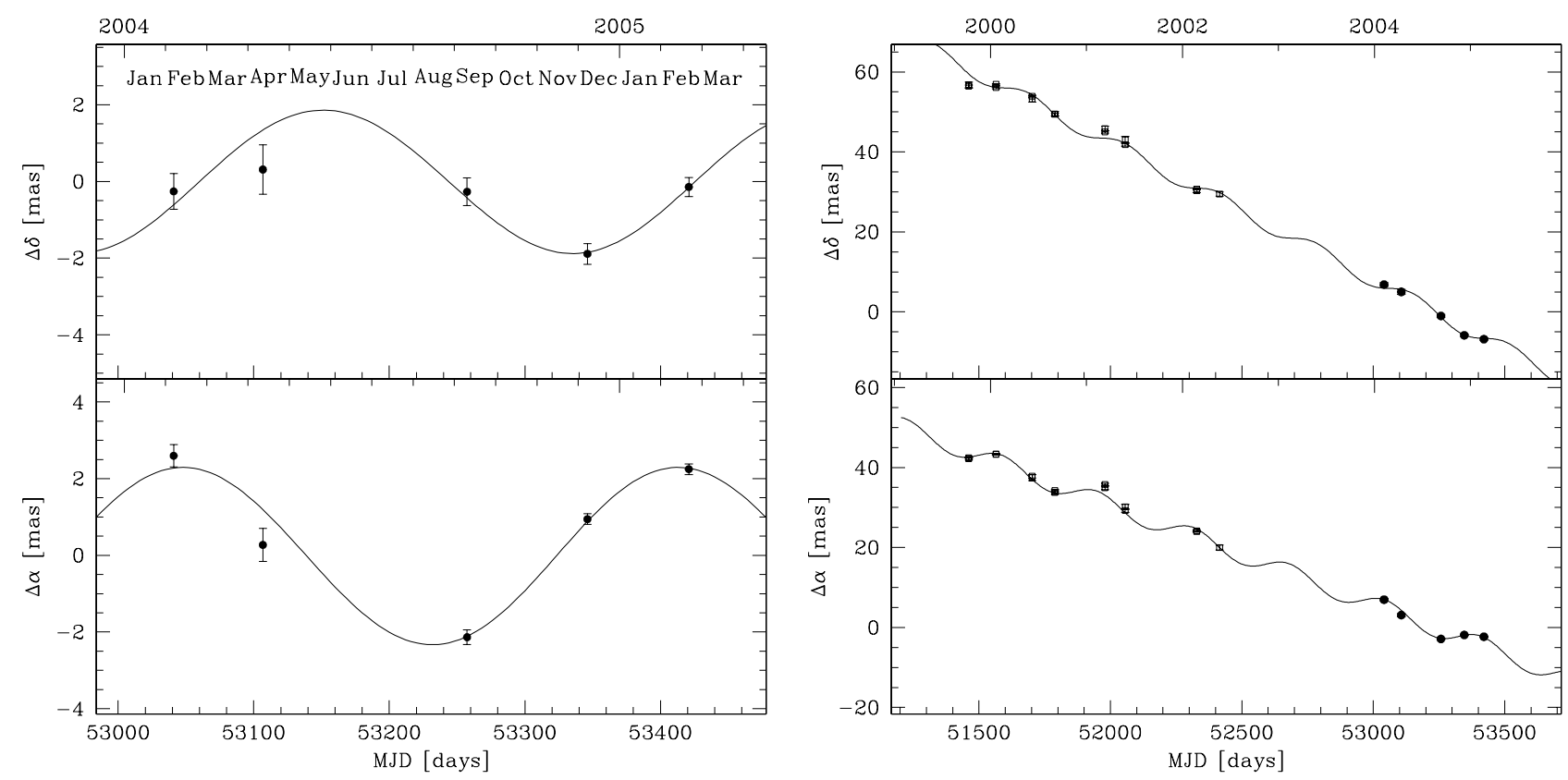

Fig. 2. Similar to Fig. 1. The position of the brightest $1667 \mathrm{MHz}$ maser spots of S CrB with respect to J1522+3144, which was used as in-beam calibrator during the 5 epochs presented here. Drawn is the best fitting parallax signature after subtracting the proper motions for the 5 new epochs with in-beam calibration (left) and the proper motion and parallax trajectory including the previous observations of the brightest 1665 and $1665 \mathrm{MHz} \mathrm{OH}$ maser spots (V03) for a total of 13 epochs (right).

observations were perfomed using the $\mathrm{NRAO}^{1}$ VLBA and the sample of stars consisted of the Mira variable stars U Her, S CrB, RR Aql, R Aql, R Cas and Y Cas. Only for the first three of these sources did we manage to detect maser emission that was strong enough to image accurately. Their periods, velocities with respect to the Local Standard of Rest (LSR), Hipparcos parallaxes and proper motions and $P-L$ distances are given in Table 1 . The

1 The National Radio Astronomy Observatory (NRAO) is a facility of the National Science Foundation operated under cooperative agreement by Associated Universities, Inc. observations of all sources were scheduled in the same observing block for a total of $12 \mathrm{~h}$. This resulted in approximately $2 \mathrm{~h}$ of observations per maser source, including the time spent on the extragalactic phase reference sources. Due to some observational problems (strong interference, telescope and recording failures), a few hours of observations at 3 of the epochs were unusable. Fortunately, none of the three detected sources (U Her, $\mathrm{S} \mathrm{CrB}$ and RR Aql) were affected more than once and we obtained 5 epochs of good data for each of them. The resulting average beam size for the observations was $14 \times 8$ mas. 

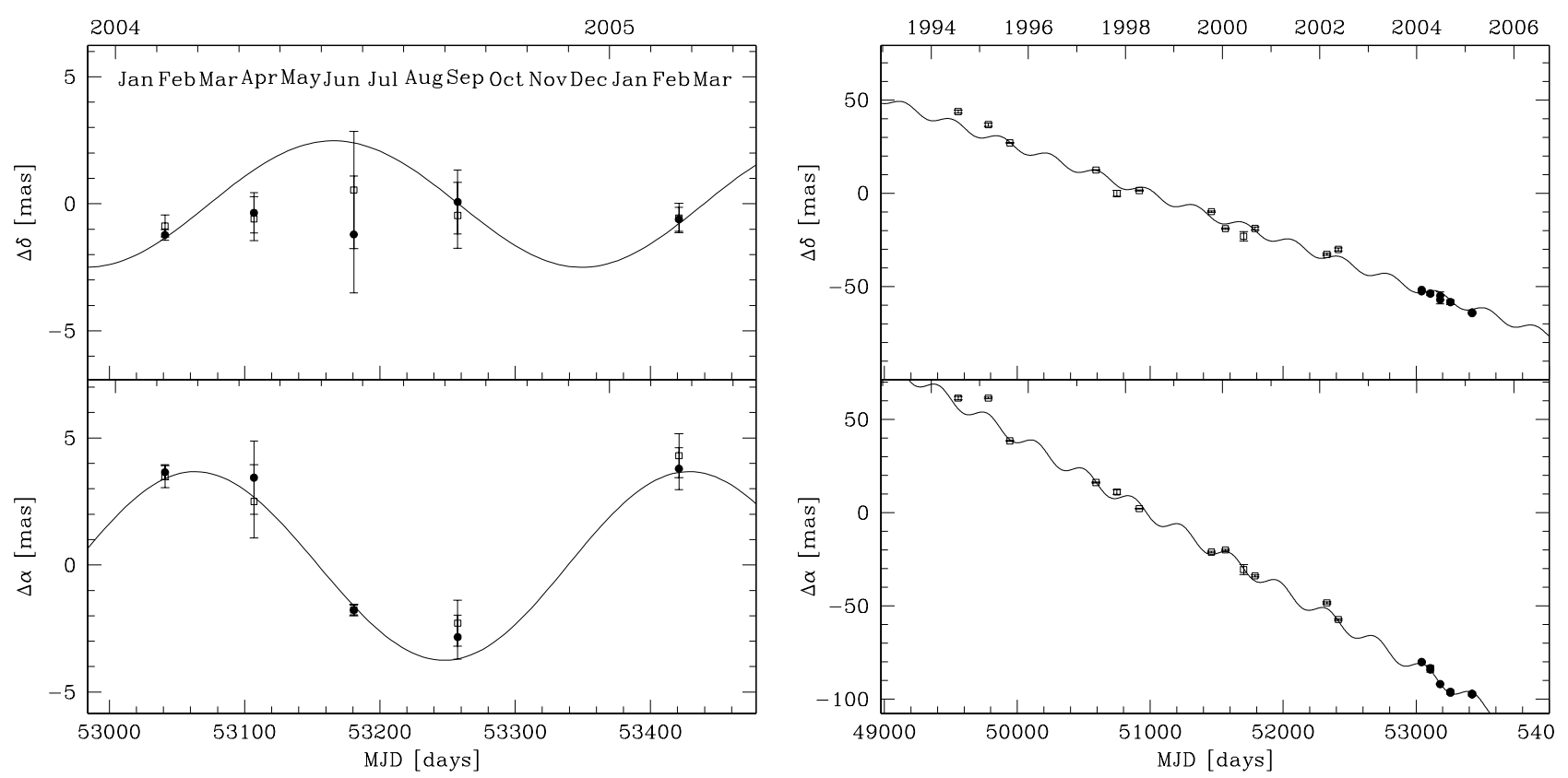

Fig. 3. Similar to Fig. 1. The position of the most blue-shifted 1665 and $1667 \mathrm{MHz}$ maser spots of U Her, that have been found to correspond to the stellar image, with respect to the primary calibrator J1630+2131. Drawn is the best fitting parallax signature after subtracting the proper motions for combining the results on both maser transitions for the 5 recent epochs of observations (left), and the proper motion and parallax trajectory combining the new observations with the 12 previous epochs (right).

Table 1. The sample.

\begin{tabular}{|c|c|c|c|c|c|c|}
\hline \multirow{2}{*}{$\begin{array}{l}\text { Source } \\
\mathrm{S} \mathrm{CrB}\end{array}$} & \multirow{2}{*}{$\begin{array}{c}\begin{array}{c}\text { Period } \\
\text { (days) }\end{array} \\
360\end{array}$} & \multirow{2}{*}{$\frac{\begin{array}{c}V_{\text {star }} \\
\left(\mathrm{km} \mathrm{s}^{-1}\right)\end{array}}{0.0}$} & \multirow{2}{*}{$\begin{array}{c}\begin{array}{c}\text { Hipparcos Parallax } \\
(\mathrm{mas})\end{array} \\
2.40 \pm 1.17^{a}\end{array}$} & \multicolumn{2}{|c|}{$\begin{array}{l}\text { Hipparcos Proper motion } \\
\text { RA, Dec (mas/yr) }\end{array}$} & \multirow{2}{*}{$\begin{array}{r}d_{P-L}{ }^{b} \\
(\mathrm{pc})\end{array}$} \\
\hline & & & & $-8.33 \pm 0.93$ & & \\
\hline U Her & 406 & -14.5 & $1.88 \pm 1.31^{a}$ & & & 380 \\
\hline RR Aql & 394 & 27.8 & $2.48 \pm 2.57$ & $-24.01 \pm 4.18$ & $-47.66 \pm 2.80$ & 540 \\
\hline
\end{tabular}

${ }^{a}$ Recalculated by Knapp et al. (2003). ${ }^{b}$ From Whitelock \& Feast (2000).

\subsection{In-beam calibration}

To improve upon the results obtained in V03, we used in-beam calibration in addition to the regular nodding phase referencing. As shown in Chatterjee et al. (2005), the astrometric accuracy that can be obtained by phase referencing is strongly dependent on the separation between the target and reference source. After initial calibration on a primary (nodding) phase reference source, residual calibration errors arise primarily from the unmodeled ionosphere between target and reference source. These errors can be significantly reduced by using an secondary calibrator in the primary telescope beam, reducing the angular throw as well as the need for time extrapolation (e.g. Fomalont et al. 1999). Such a secondary in-beam calibrator can be much fainter than the primary calibrator, with a flux $\gtrsim 10 \mathrm{mJy}$. In an effort to find in-beam calibrators with sufficient flux that additionally are suitably compact at VLBA baselines, we observed the brightest NVSS sources (Condon et al. 1998) near a number of maser stars with the Very Large Array (VLA) at $8.4 \mathrm{GHz}$. In addition to the stars making up the sample presented here, we also observed possible calibrator sources near W Hya and RT Oph. In total 40 sources were observed for $3 \mathrm{~min}$ each. These were then reduced using AIPS without any special processing. The images of all the sources, including those that were not detected, are presented in Figs. 6-9 in the electronic form. There we also present Table 3 with positions and fluxes extracted using the AIPS JMFIT task. From the VLA observations we determined the best VLBA in-beam candidates. No good candidates were found for W Hya and RT Oph and also for U Her there was no obvious good in-beam candidate. However, as U Her is the longest astrometrically monitored $\mathrm{OH}$ maser source, it was still included in our sample even though in-beam calibration turned out to be impossible. As in V03, we used J1630+2131 with a separation of $2.8^{\circ}$ as primary calibrator for U Her. For S CrB, the strongest in-beam calibrator source was also the primary calibrator $(\mathrm{J} 1522+3144)$ used in V03 at a distance of $24^{\prime}$, so we were able to accurately calibrate the masers of $\mathrm{S} \mathrm{CrB}$ without nodding the telescopes. For RR Aql we used both a primary calibrator (J2015-0137 at 4.4 from RR Aql) and an in-beam calibrator (NVSS J195655-013615), with a target-calibrator separation of $\sim 20^{\prime}$ (see Table 3 in the on-line material for detailed coordinates).

\subsection{Data correlation}

We used a mixed bandwidth setting to observe both the $1667 \mathrm{MHz} \mathrm{OH}$ maser line and the continuum calibrator simultaneously. One frequency band with a bandwidth of $500 \mathrm{kHz}$ was centered on the stellar velocity and correlated with moderate spectral resolution $\left(1.95 \mathrm{kHz}=0.36 \mathrm{~km} \mathrm{~s}^{-1}\right)$. Simultaneously three $4 \mathrm{MHz}$ wide bands were recorded to detect the continuum reference sources, with one of the bands also covering the $1665 \mathrm{MHz} \mathrm{OH}$ line. These bands were correlated twice with a 
spectral resolution of $15.6 \mathrm{kHz}$, equaling $2.88 \mathrm{~km} \mathrm{~s}^{-1}$. The first of the wide band correlator passes used the position of the inbeam reference source for calibration purposes, while the second pass used the stellar position to allow us to detect the $1665 \mathrm{MHz}$ $\mathrm{OH}$ maser line.

\subsection{Data calibration}

The astrometric VLBA data was calibrated using AIPS, with amplitude calibration based on the antenna system temperatures and phase, rate and delay calibration based on the primary and in-beam calibrators. We applied ionospheric corrections using the AIPS task TECOR, but found that these do not significantly improve our data quality. Additionally, we used CLCOR to correct for errors in the Earth Orientation Parameters (EOP) that affected VLBA observations between May 2003 and August 2005 (Walker et al. 2005). For a consistent solution we determined, when possible a model of the primary and available in-beam calibrators using all epochs of data. As the primary calibrator $(\mathrm{J} 1630+2131)$ of $U$ Her showed structural changes between the different epochs, this forced us to construct independent calibrator models for every epoch. The models were then used to iteratively calibrate the data.

As the calibration solutions from both the primary and the inbeam calibrators are determined on the wide-band $(3 \times 4 \mathrm{MHz})$ data, special care needed to be taken connecting these solutions to the narrow-band spectral line data. A special task written in AIPS for the previous observations presented in vL00 and V03 was rewritten in the ParselTongue scripting language (Kettenis et al. 2006) and was then used to transfer the calibration solutions.

\subsection{Error analysis}

For the two sources with in-beam calibrators $(\mathrm{S} \mathrm{CrB}$ and RR Aql), the astrometric errors on the observational data points were taken to be the uncertainty in calibrator and maser feature position added in quadrature. The uncertainty in calibrator position is determined using Beam/[2 $\left.\times S N R_{\mathrm{c}}\right]$ for unresolved calibrator sources, while that of the maser feature and of the resolved calibrator sources is $\theta_{\mathrm{c}, \mathrm{m}} /\left[2 \times S N R_{\mathrm{c}, \mathrm{m}}\right]$ with $S N R_{\mathrm{c}, \mathrm{m}}$ the signal-to-noise ratio on the calibrator and maser and $\theta_{\mathrm{c}, \mathrm{m}}$ the full width half-maximum size of the calibrator and the maser feature respectively. This size is equal to the Beam-size when the maser is unresolved, which is typically the case. The total positional error is of the order of 0.2 mas during the best observing conditions, with the error as a result of the uncertainty in calibrator position typically smallest by an order of magnitude. The total error increases to $\sim 0.7$ mas for the weakest masercalibrator combinations and the worst ionospheric conditions. The here described error estimates produced least $\chi^{2}$-fits with reduced $\chi^{2}=1.01$ and 1.86 for 5 degrees of freedom for RR Aq1 and $\mathrm{S} \mathrm{CrB}$ respectively.

For U Her, where no suitable in-beam calibrator was found, astrometric errors are expected to be larger. Fortunately, we were able to detect both 1665 and $1667 \mathrm{MHz}$ OH masers. As was found in vL00, the most blue-shifted maser features in both transititions are coincident, and correspond to the amplified stellar image. We thus estimate an additional factor in the positional uncertainty from the internal scatter between the 1665 and $1667 \mathrm{MHz}$ most blue-shifted maser features. This additional scatter factor, of the order of $\sim 0.6$ mas, was also added quadratically to the positional error estimate described above, producing

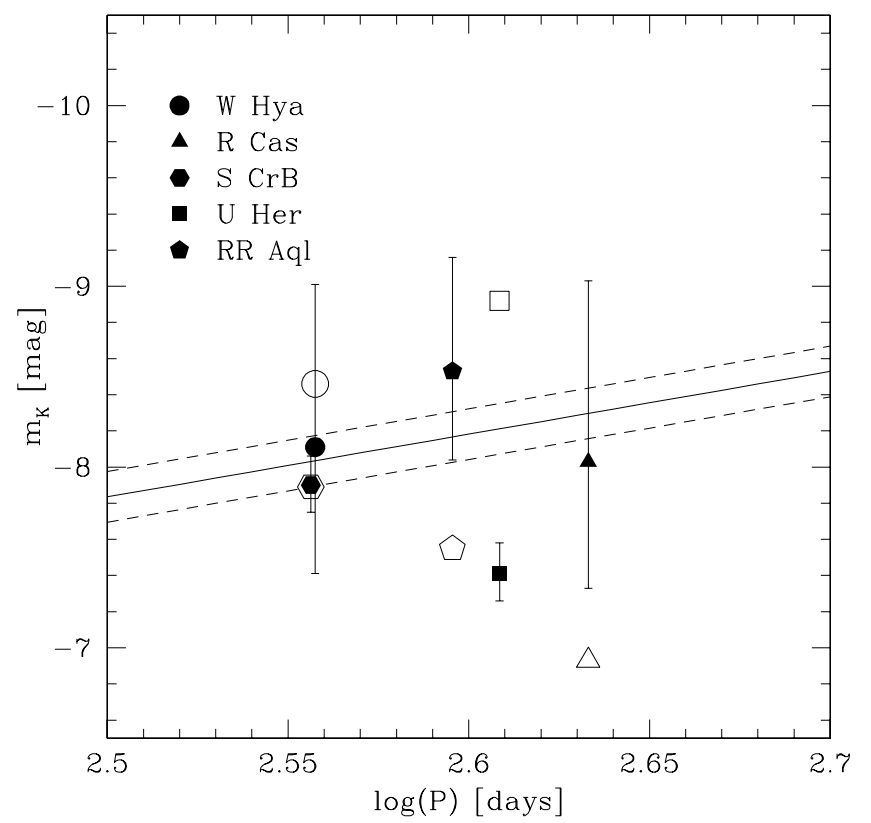

Fig. 4. Period vs. $K_{0}$ magnitudes for the $\mathrm{OH}$ maser stars with VLBI interferometric distances, including those from V03. The solid symbols and the error bars are determined using the VLBI distances. The open symbols are the values using the Hipparcos distances. The solid line is the $P-L$ relation determined by Whitelock \& Feast (2000) on the oxygen rich Mira stars observed with Hipparcos, the dashed lines are the spread in the relation due to the error in the $P-L$ relation zero-point.

a typical error of $\sim 0.7$ mas. As a result, the $\chi^{2}$-fit to both 1667 and $1665 \mathrm{MHz} \mathrm{OH}$ masers of $\mathrm{U}$ Her simultaneously, has a reduced $\chi^{2}=1.61$ for 13 degrees of freedom. These $\chi^{2}$ results imply that the error estimates for all three of the sources are a fairly good representation of the true astrometric errors.

Finally, we have checked the robustness of the astrometric results by randomly removing individual data points and performing new fits on the reduced data set. We find that for $\mathrm{S} \mathrm{CrB}$ and RR Aql the fit solutions are consistent within $1 \sigma$. For U Her the solutions are consistent within $\sim 1.2 \sigma$, hinting at a slight underestimate of the fitting errors for $\mathrm{U}$ Her.

\section{Results}

The results of the $\chi^{2}$-fits are presented in Table 2 . The table contains the parallax and proper motion results derived from the recent observational epochs as well as, for comparison, the results from V03 for U Her and S CrB. For these sources we have also performed a fit combining the entire data-set, allowing for an additional zero-position off-set between the earlier epochs and the data presented here. Such an additional off-set is needed because of changes in the calibrator situation and since, especially for $\mathrm{S} \mathrm{CrB}$, we might be tracing a new maser feature. Additionally, the errors for the early epochs in the combined fitting have been increased by a factor of two, as in V03 only the formal position fitting errors were used while in the original scheme the ionospheric contributions could not be easily estimated. The table gives the velocity $V_{\mathrm{LSR}}$ of the maser feature used for the fit with respect to the Local Standard of Rest (LSR) as well as which maser transition (1665 or $1667 \mathrm{MHz}$ ) was used for the fit. 
Table 2. Results.

\begin{tabular}{lcccccccc}
\hline \hline Source & $\begin{array}{c}\text { Maser } \\
(\mathrm{MHz})\end{array}$ & Note $^{a}$ & $\begin{array}{c}\text { VLBI parallax } \\
(\mathrm{mas})\end{array}$ & \multicolumn{2}{c}{$\begin{array}{c}\text { VLBI proper motion } \\
\text { RA, Dec (mas/yr) }\end{array}$} & \# epochs & $\begin{array}{c}V_{\mathrm{LSR}}{ }^{b} \\
\left(\mathrm{~km} \mathrm{~s}^{-1}\right)\end{array}$ & $\begin{array}{c}d_{\text {VLBI }} \\
(\mathrm{pc})\end{array}$ \\
\hline S CrB & 1667 & & $2.39 \pm 0.17$ & $-8.58 \pm 0.38$ & $-13.21 \pm 0.61$ & 5 & $4.6 \mathrm{~km} \mathrm{~s}^{-1}$ & $418_{-18}^{+21}$ \\
& both & A & $2.31 \pm 0.33$ & $-9.08 \pm 0.27$ & $-12.49 \pm 0.33$ & 8 & $3.2 / 2.9 \mathrm{~km} \mathrm{~s}^{-1}$ & \\
& both & B & $2.36 \pm 0.23$ & $-9.06 \pm 0.23$ & $-12.52 \pm 0.29$ & $5+8$ & & \\
\hline U Her & both & & $3.76 \pm 0.27$ & $-16.99 \pm 0.77$ & $-11.88 \pm 0.50$ & 5 & $-20.2 \mathrm{~km} \mathrm{~s}^{-1}$ & $266_{-28}^{+32}$ \\
& 1667 & A & $3.61 \pm 1.04$ & $-14.94 \pm 0.38$ & $-9.17 \pm 0.42$ & 12 & $-20.4 \mathrm{~km} \mathrm{~s}^{-1}$ & \\
& both & B & $3.74 \pm 0.61$ & $-14.98 \pm 0.29$ & $-9.23 \pm 0.32$ & $5+12$ & & \\
\hline RR Aql & 1665 & & $1.58 \pm 0.40$ & $-25.11 \pm 0.74$ & $-49.82 \pm 0.54$ & 5 & $24 \mathrm{~km} \mathrm{~s}^{-1}$ & $633_{-128}^{+214}$ \\
\hline
\end{tabular}

${ }^{a}$ A: results from V03; B: results from combined fit including V03 epochs.

${ }^{b}$ LSR Velocity of fitted maser features.

\subsection{RR Aql}

RR Aql is one of the new sources added to the sample, for which Hipparcos did not manage to obtain a significant parallax. We did not manage to detect any $1667 \mathrm{MHz}$ maser emission, but were fortunately able to image a $\sim 0.1 \mathrm{Jy} /$ beam $1665 \mathrm{MHz}$ maser feature in the low spectral resolution data. The feature was found at $24 \mathrm{~km} \mathrm{~s}^{-1}$, blue-shifted with respect to the stellar velocity, but at a velocity unlikely to belong to an amplified stellar image. We managed to detect and image the feature at 5 epochs with respect to the nearby in-beam calibrator. This yielded the smallest parallax determined to date for $\mathrm{OH}$ maser astrometry, even though the errors are dominated by the fact that the maser is weak and is only detected in the low resolution spectral line data-set.

\section{2. $S \mathrm{CrB}$}

S CrB was previously monitored with the VLBA (V03), at which time we were able to detect bright masers at both 1665 and $1667 \mathrm{MHz}$ (at $V_{\mathrm{LSR}}=3.2$ and $2.9 \mathrm{~km} \mathrm{~s}^{-1}$ respectively). In the new epochs we were only able to detect a $1667 \mathrm{MHz}$ maser feature, red-shifted with respect to the stellar velocity, at $V_{\mathrm{LSR}}=4.6 \mathrm{~km} \mathrm{~s}^{-1}$. The feature was fairly weak during the first 2 epochs, with the flux decreasing from $0.07 \mathrm{Jy} /$ beam in the first to below $0.05 \mathrm{Jy} /$ beam in the second epoch. The third epoch turned out to be unusable, but during the last three epochs the maser had brightened again to $\sim 0.11 \mathrm{Jy} /$ beam. Thus, the astrometric errors were almost a factor of two smaller in the last three epochs. Both the parallax and proper motion fit results from the newest epochs are fully consistent with the result obtained from the earlier epochs.

\section{3. $U$ Her}

$\mathrm{U}$ Her is the $\mathrm{OH}$ maser source which has been the target of the longest astrometric VLBI monitoring campaign, and currently has data spanning almost 12 years (vL00, V03). In the new observations, we again detected the most blue-shifted maser feature corresponding to the amplified stellar image in both the 1665 and $1667 \mathrm{MHz}$ maser transition, with a flux varying between 0.8 and $1.1 \mathrm{Jy} / \mathrm{beam}$. In addition, we detected a number of weaker features which are discussed in Sect. 4.2. While the parallax is also fully consistent with the earlier determined parallax, albeit with a significantly smaller error, the proper motion has changed considerably. As was already found in V03, the calibrator used for phase referencing, J1630+2131, shows structural changes on timescales at the order of several months. This is possibly the cause of the differences in proper motions. Figure 10 of the online material shows a series of images of J1630+2131 for each observational epoch indicating these changes. As described in V03, the internal calibrator motions are expected to be relatively slow compared to the annual timescale of the parallax and we thus think it unlikely that this will bias the parallax measurement. However, the motions will introduce additional scatter and some caution has to be taken with respect to the quoted uncertainties. Because of the proper motion difference, caution has to be taken in using the fitted motion resulting from the combined epochs fitting.

\section{Discussion}

\subsection{Comparisson with previous results}

The distances to the $\mathrm{OH}$ bearing Mira variable stars $\mathrm{S} \mathrm{CrB}$ and U Her derived in this paper are fully consistent with the previously published values in V03. The uncertainty on the parallax measurement decreased by over a factor of 2 , even with significantly fewer observational epochs, illustrating the astrometric improvements. The errors on the proper motion measurements are larger, mainly due to the shorter time-baseline of the new observations. The most accurate proper motions have therefore been obtained by simultaneously fitting the maser positions from both the current and previous observational epochs as described above.

The astrometric measurements are also a significant improvement on the Hipparcos derived values, which suffered from the fact that the stars in our sample are relatively faint, variable, AGB stars (see V03 for more details). When comparing the VLBI derived distances in Table 2 with distances derived from a $P-L$ relation in Table 1, we find that, while for both $\mathrm{S} \mathrm{CrB}$ and RR Aql the distances are reasonably consistent, the U Her VLBI distance is significantly lower. This can also be seen in Fig. 4, which shows position of the OH maser stars with VLBI parallaxes in the $P-L$ diagram. As was speculated in vL00 and V03, the discrepancy for U Her could be a result from U Her pulsating in the fundamental mode, while the other stars are pulsating in the first overtone mode. However, as it has been argued that all Mira stars are pulsating in the fundamental mode (Wood 1998), the offset of U Her more likely highlights the scatter in the $P$ $L$ relation or the uncertainty in individual magnitude determinations. We rule out that the offset is introduced by the absence of an in-beam calibrator for this source, because we can imagine no systematic effect that can bias the parallax to larger values over all the years we have monitored the source. 

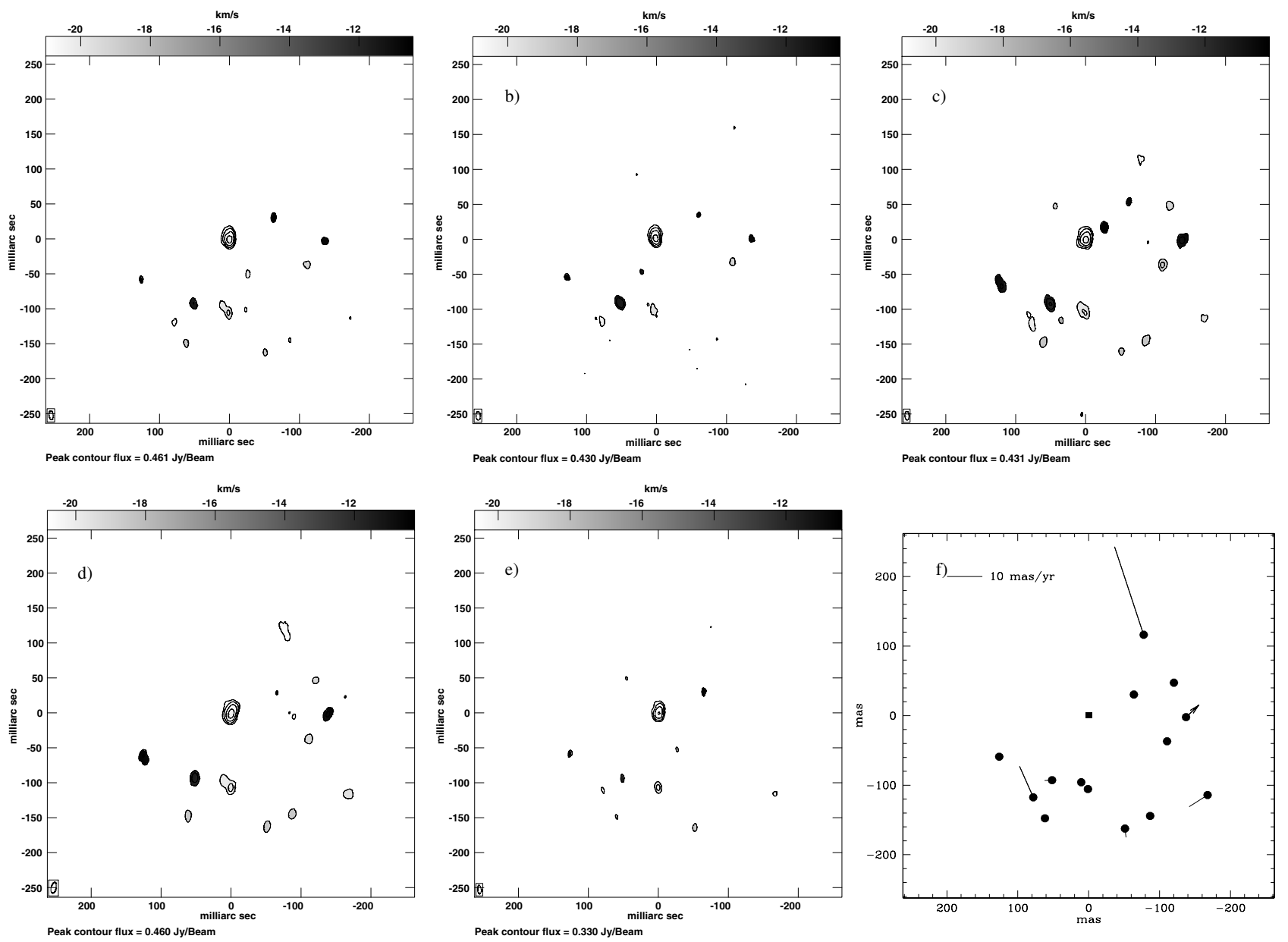

Fig. 5. a)-e) Images for 5 epochs of the $1667 \mathrm{MHz} \mathrm{OH}$ maser emission of U Her referenced to the most brightest maser. The brightest and most blue-shifted maser feature is prominent in all images and is identified with the stellar image amplified by the masing material at the front side of the expanding shell (V03). The greyscale indicates the first moment map with the velocity scale indicated at the top of the figures. The contours indicate the total intensity $I$, drawn at levels of 40,80,160 and $320 \mathrm{mJy} /$ Beam. The rms noise varies somewhat for the different epochs but is $\sim 12 \mathrm{mJy} / \mathrm{Beam}$. The peak intensity is given for each individual image. f) The maser features that were observed during at least 2 epochs with vectors indicating their proper motion. The arrow denotes a $3 \sigma$ proper motion detection, no significant proper motion was detected for any of the other features but for illustration purposes we show $1-3 \sigma$ proper motions with the regular vectors. The solid square indicates the position of the stellar image.

\subsection{Morphology and internal motions of the $\mathrm{OH}$ masers of $U$ Her}

While for S CrB and RR Aql we only detect maser features in a few neighbouring red-shifted and blue-shifted spectral channels respectively, we observe a large number of both blue- and red-shifted features for U Her. Figure 5a-e show the first moment greyscale maps with overplotted total intensity contours for the 5 succesful U Her epochs. The maps are all referenced the most blue-shifted maser feature that has been identified as the stellar image. Several of the maser features are detected in multiple epochs and these are shown in Fig. 5f. The brightest red-shifted masers are all detected with $V_{\mathrm{LSR}}$ between -10 and $-12 \mathrm{~km} \mathrm{~s}^{-1}$ while the blue-shifted masers are located at $V_{\mathrm{LSR}}$ between -17 and $-21 \mathrm{~km} \mathrm{~s}^{-1}$. There is no obvious difference in the extent of the red- and blue-shifted maser features, which are both spread over $\sim 200 \times 200$ mas, corresponding to $\sim 55 \times 55 \mathrm{AU}$ at the $266 \mathrm{pc}$ distance of U Her. Figure 5 does show that the maser distribution is distinctly asymmetrical with respect to the stellar image, with hardly any maser features detected to the North-East. This is significantly different from the 1665 and $1667 \mathrm{GHz}$ maser distribution observed in 1984 with MERLIN
(Chapman et al. 1994), but similar to our previous 1665 and $1667 \mathrm{GHZ} \mathrm{OH}$ maser observations between 1995 and 2002

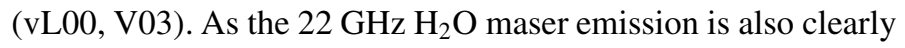
weakest towards the North-East (Vlemmings et al. 2002; Bains et al. 2003), the observed non-spherical maser distribution is likely due to a real asymmetry in the CSE.

As several U Her maser features, besides the stellar image, can be traced over multiple epochs, we are able to study the internal motions in the maser envelope. One red-shifted maser feature, at $V_{\mathrm{LSR}}=-11.51 \mathrm{~km} \mathrm{~s}^{-1}$, was persistent over the entire year of observation, while three maser features are found in 4 epochs. A further 5 features can be traced over three epochs while another 5 are detected in only 2 epochs. Although none of these features are bright enough to be included in the regular astrometric fitting, we are able to test the consistency of our observations by determining the motions of these features after self-calibrating on the blue-shifted maser feature amplifying the stellar emission. By applying the self-calibration we remove the stellar proper motion and parallax, after which we fit for residual proper motions of the maser features. For most of the maser features we do not detect significant proper motion. Only for one red-shifted maser feature, that was detected in the 
first three epochs at $V_{\mathrm{LSR}}=-10.81 \mathrm{~km} \mathrm{~s}^{-1}$, we detect a proper motion of $\mu=5.1 \pm 1.5$ mas $\mathrm{yr}^{-1}$, which corresponds to a velocity of $6.4 \pm 1.9 \mathrm{~km} \mathrm{~s}^{-1}$ at the distance to U Her. Assuming a spherically expanding shell, this implies, from the angular off-set to the stellar image and the radial velocity, an outflow velocity of $\sim 7.5 \mathrm{~km} \mathrm{~s}^{-1}$ in the $\mathrm{U}$ Her $\mathrm{OH}$ maser region. The proper motion of this feature is indicated with a thick line in Fig. 5. Performing a weighted ensemble average of the masers in three quadrants $(\alpha<0$ and $\delta>0, \alpha<0$ and $\delta<0, \alpha>0$ and $\delta<0)$ reveals no significant motions. Considering that we only observe the front and back side of the maser shell this is not surprising, as the outflow will be predominantly in the radial direction. Thus, as no residual proper motion is detected, the motion determined from the astrometric fitting to the amplified stellar image can be taken as the true stellar proper motion, with only a possible contribution from the internal motions of the phase reference source.

As we have concluded that the majority of the maser spots show no significant proper motion when referencing them to the stellar image, the residual motion of the maser features is likely due to a combination of the intrinsic astrometric errors and turbulent motions in the masing medium. We find that the rms scatter of the masers detected at multiple epochs is $\sigma_{\text {pos }}=$ $2.0 \pm 1.3$ mas. Taking into account the intrinsic positional fitting uncertainty, including the error discussed in Sect. 2.4, which for these weak maser features is of the order of 1.5 mas, small but significant residual motions are present. These we attribute to turbulence at the level of $1-2 \mathrm{~km} \mathrm{~s}^{-1}$, only slightly higher than the typical assumed value of $1 \mathrm{~km} \mathrm{~s}^{-1}$ (Diamond et al. 1985).

\section{Concluding remarks}

We have been able to significantly improve the parallax measurements for a number of $\mathrm{OH}$ maser stars. The improvements were the results of using nearby phase reference sources in the form of in-beam calibrators, and generally good observing conditions due to a minimum in solar activity. As a result the parallax uncertainties were decreased by up to a factor of $\sim 4$ to the order of $\sim 0.2$ mas, using only 5 observing epochs compared to the $8-12$ epochs presented in V03. Thus, we have convincingly shown the capability of VLBI astrometry to determine distances up to $1 \mathrm{kpc}$ to AGB stars using their circumstellar $\mathrm{OH}$ masers, even when no special maser feature amplifying the stellar image is found. The limit on distance measurements could potentially be stretched to $\sim 2 \mathrm{kpc}$ or $\pi \sim 0.5$ mas when the $\mathrm{OH}$ maser is strong $(\gtrsim 0.25 \mathrm{Jy} /$ beam $)$ and a bright compact in-beam calibrator is available. The precision will be further increased when a number of different maser features can be traced over a sufficient number of epochs. This opens up the possibility to use
$\mathrm{OH}$ maser astrometry to determine the parallaxes to a large number of $\mathrm{OH}$ maser stars with previously unknown distances, which can for instance be used to contruct a $P-L$ relation for the most heavily enshrouded AGB stars.

Acknowledgements. This research was partly supported by a Marie Curie IntraEuropean fellowship within the 6th European Community Framework Program under contract number MEIF-CT-2005-010393. WV thanks the Max-Planck Institute for Astronomy in Heidelberg for the regular hospitality. We acknowledge the effort of Daniele Biancu for implementing the mixed bandwidth calibration scheme in ParselTongue. ParselTongue is developed within the context of the RadioNet Joint Research Activity ALBUS. This work has benefited from research funding from the European Community's sixth Framework Programme under RadioNet R113CT 20035058187.

\section{References}

Bains, I., Cohen, R. J., Louridas, A., et al. 2003, MNRAS, 342, 8

Brisken, W. F., Benson, J. M., Goss, W. M., \& Thorsett, S. E. 2002, ApJ, 571, 906

Chapman, J. M., Sivagnanam, P., Cohen, R. J., \& Le Squeren, A. M. 1994, MNRAS, 268, 475

Chatterjee, S., Vlemmings, W. H. T., Brisken, W. F., et al. 2005, ApJ, 630, L61

Condon, J. J., Cotton, W. D., Greisen, E. W., et al. 1998, AJ, 115, 1693

Diamond, P. J., Norris, R. P., Rowland, P. R., Booth, R. S., \& Nyman, L.-A. 1985, MNRAS, 212, 1

Fomalont, E. B., Goss, W. M., Beasley, A. J., \& Chatterjee, S. 1999, AJ, 117, 3025

Hachisuka, K., Brunthaler, A., Menten, K. M., et al. 2006, ApJ, 645, 337

Honma, M., Fujii, T., Hirota, T., et al. 2003, PASJ, 55, L57

Kettenis, M., van Langevelde, H. J., Reynolds, C., \& Cotton, B. 2006, in Astronomical Data Analysis Software and Systems XV, ed. C. Gabriel, C. Arviset, D. Ponz, \& S. Enrique, ASP Conf. Ser., 351, 497

Knapp, G. R., Pourbaix, D., Platais, I., \& Jorissen, A. 2003, A\&A, 403, 993

Kurayama, T., Sasao, T., \& Kobayashi, H. 2005, ApJ, 627, L49

Mennesson, B., Perrin, G., Chagnon, G., et al. 2002, ApJ, 579, 446

Norris, R. P., Booth, R. S., Diamond, P. J., et al. 1984, MNRAS, 208, 435

Perryman, M. A. C., Lindegren, L., Kovalevsky, J., et al. 1997, A\&A, 323, L49

Sivagnanam, P., Le Squeren, A. M., Biraud, F., \& Diamond, P. J. 1990, A\&A, 229,171

van Langevelde, H. J., Vlemmings, W., Diamond, P. J., Baudry, A., \& Beasley, A. J. 2000, A\&A, 357, 945

Vlemmings, W. 2002, Ph.D. Thesis, Leiden University

Vlemmings, W. H. T., van Langevelde, H. J., \& Diamond, P. J. 2002, A\&A, 393, L33

Vlemmings, W. H. T., van Langevelde, H. J., Diamond, P. J., Habing, H. J., \& Schilizzi, R. T. 2003, A\&A, 407, 213

Walker, R. C., Dhawan, V., Brisken, W., et al. 2005, NRAO VLBA Test Memo, 69

Whitelock, P., \& Feast, M. 2000, MNRAS, 319, 759

Wittkowski, M., Boboltz, D. A., Driebe, T., \& Ohnaka, K. 2005, Mem. Soc. Astron. It., 76, 457

Wood, P. R. 1998, in Fundamental Stellar Properties, ed. T. R. Bedding, A. J. Booth, \& J. Davis, IAU Symp., 189, 299

Xu, Y., Reid, M. J., Zheng, X. W., \& Menten, K. M. 2006, Science, 311, 54 
W. H. T. Vlemmings and H. J. van Langevelde: Improved VLBI astrometry of OH maser stars, Online Material p 1

\section{Online Material}


W. H. T. Vlemmings and H. J. van Langevelde: Improved VLBI astrometry of OH maser stars, Online Material p 2
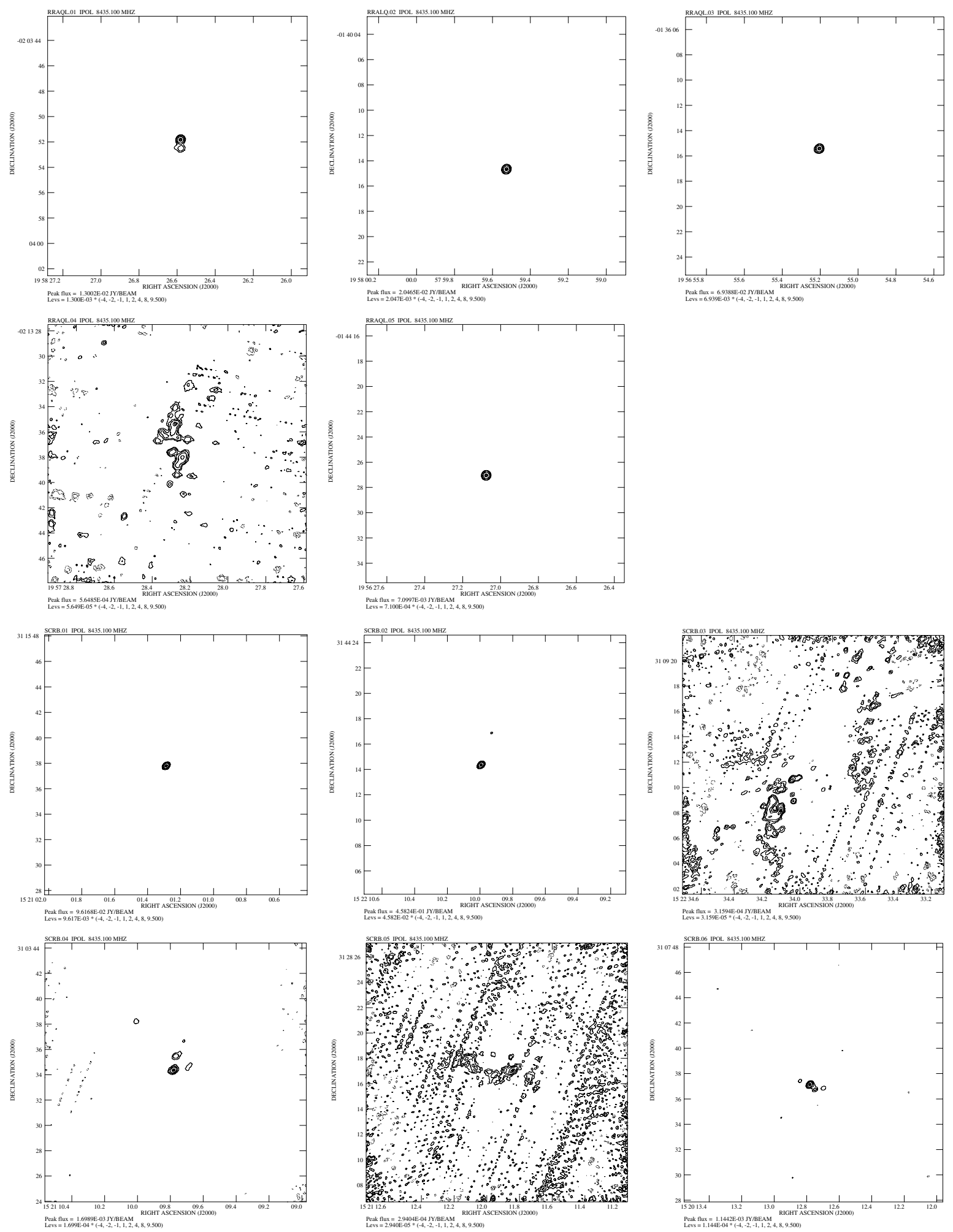

Fig. 6. Results from the $8.4 \mathrm{GHz}$ VLA in-beam calibrator search for RR Aql and S CrB. The images are labelled according to Table 3 and include non-detections for completeness. No special calibration to improve the images was performed. Peak flux and contour levels are given for each image individually. For RR Aql and S CrB, VLBA in-beam calibration was sucessfully performed on the sources RRAQL.03 and SCRB.02. 
W. H. T. Vlemmings and H. J. van Langevelde: Improved VLBI astrometry of $\mathrm{OH}$ maser stars, Online Material p 3
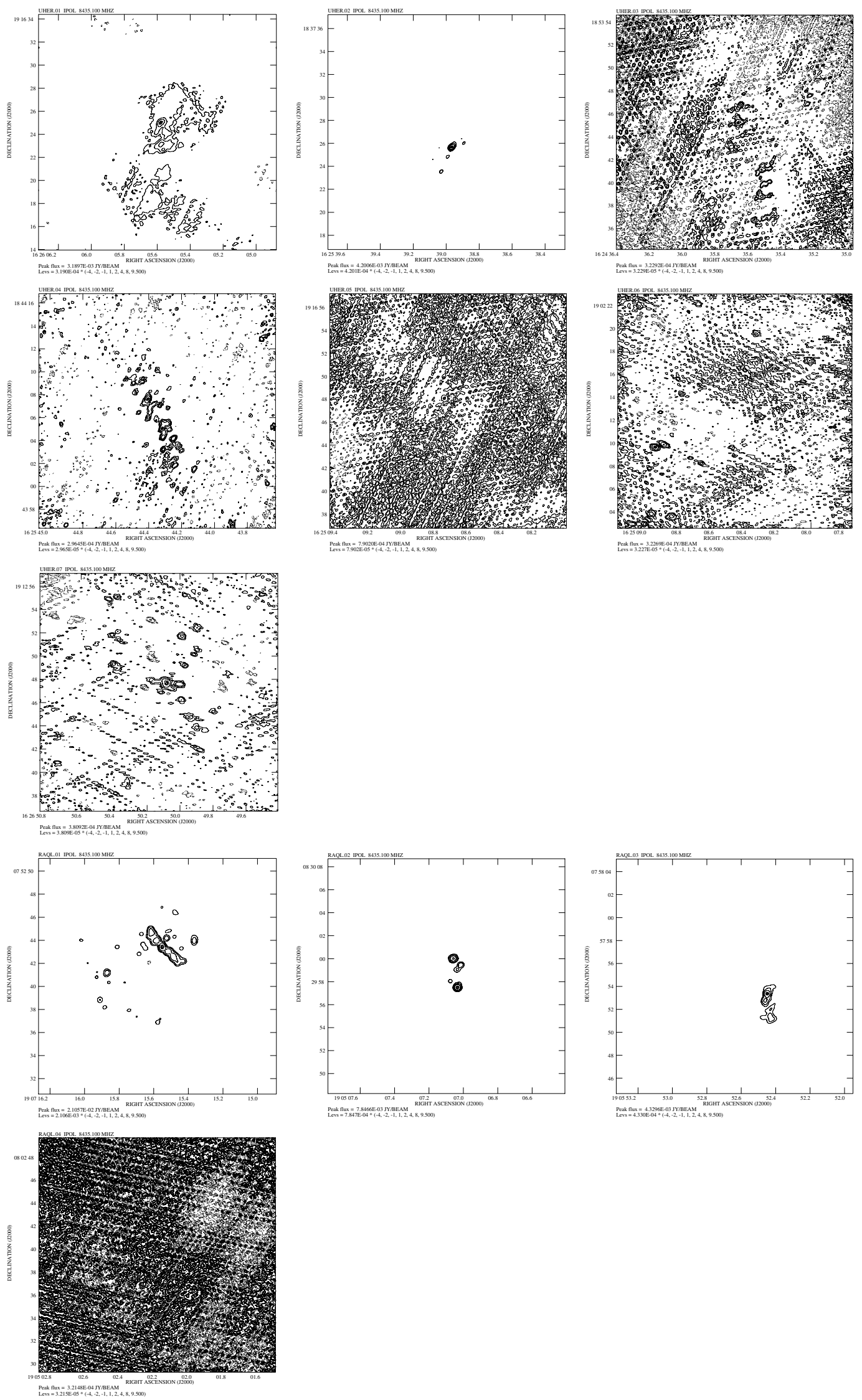

Fig. 7. As Fig. 6 for U Her and R Aql. VLBA in-beam calibration was unsuccesfully attempted on UHER.02. No masers were detected for R Aql during the VLBA observations. 
W. H. T. Vlemmings and H. J. van Langevelde: Improved VLBI astrometry of OH maser stars, Online Material p 4
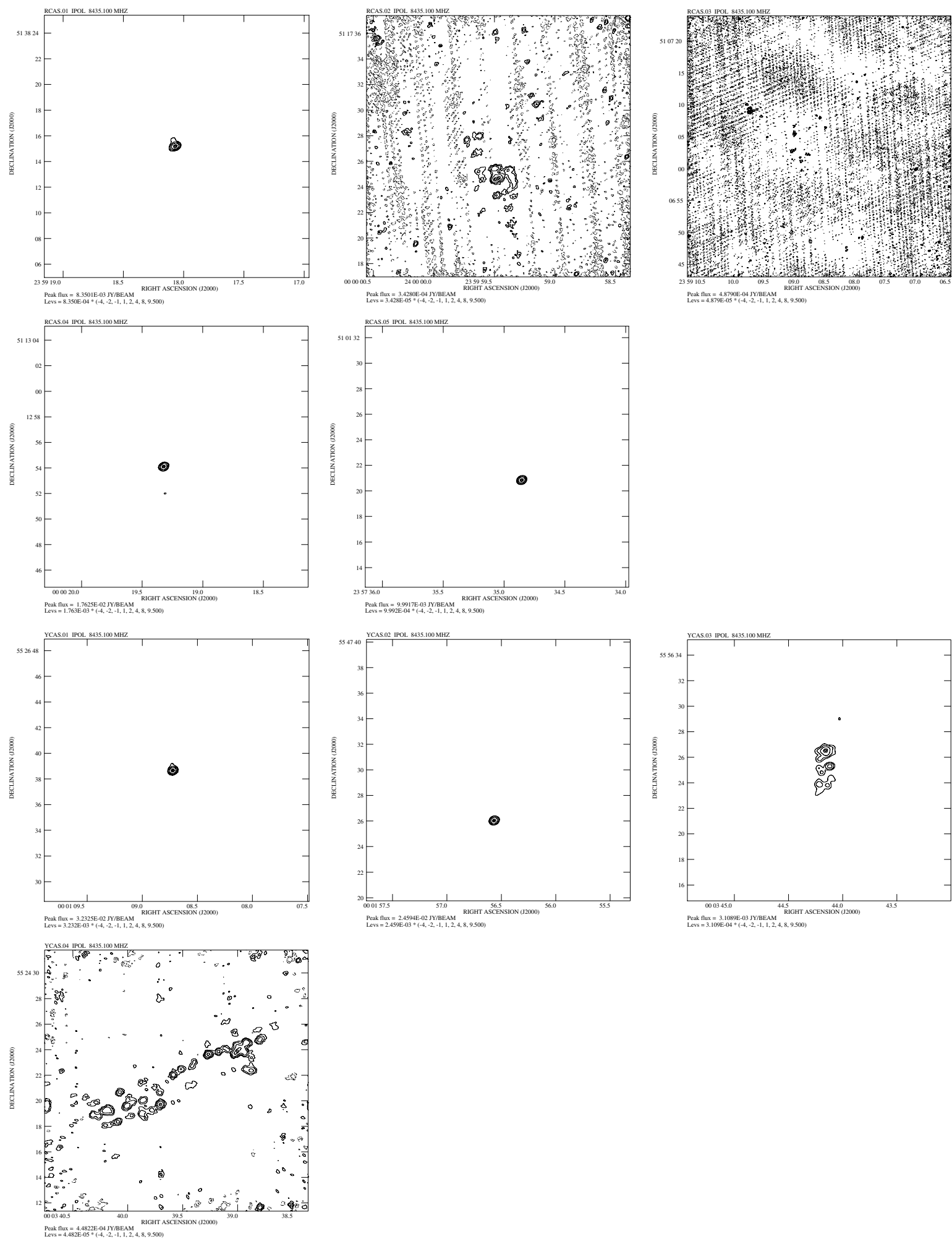

Fig. 8. As Fig. 6 for R Cas and Y Cas. No masers were detected during the VLBA observations for either star. 
W. H. T. Vlemmings and H. J. van Langevelde: Improved VLBI astrometry of $\mathrm{OH}$ maser stars, Online Material p 5
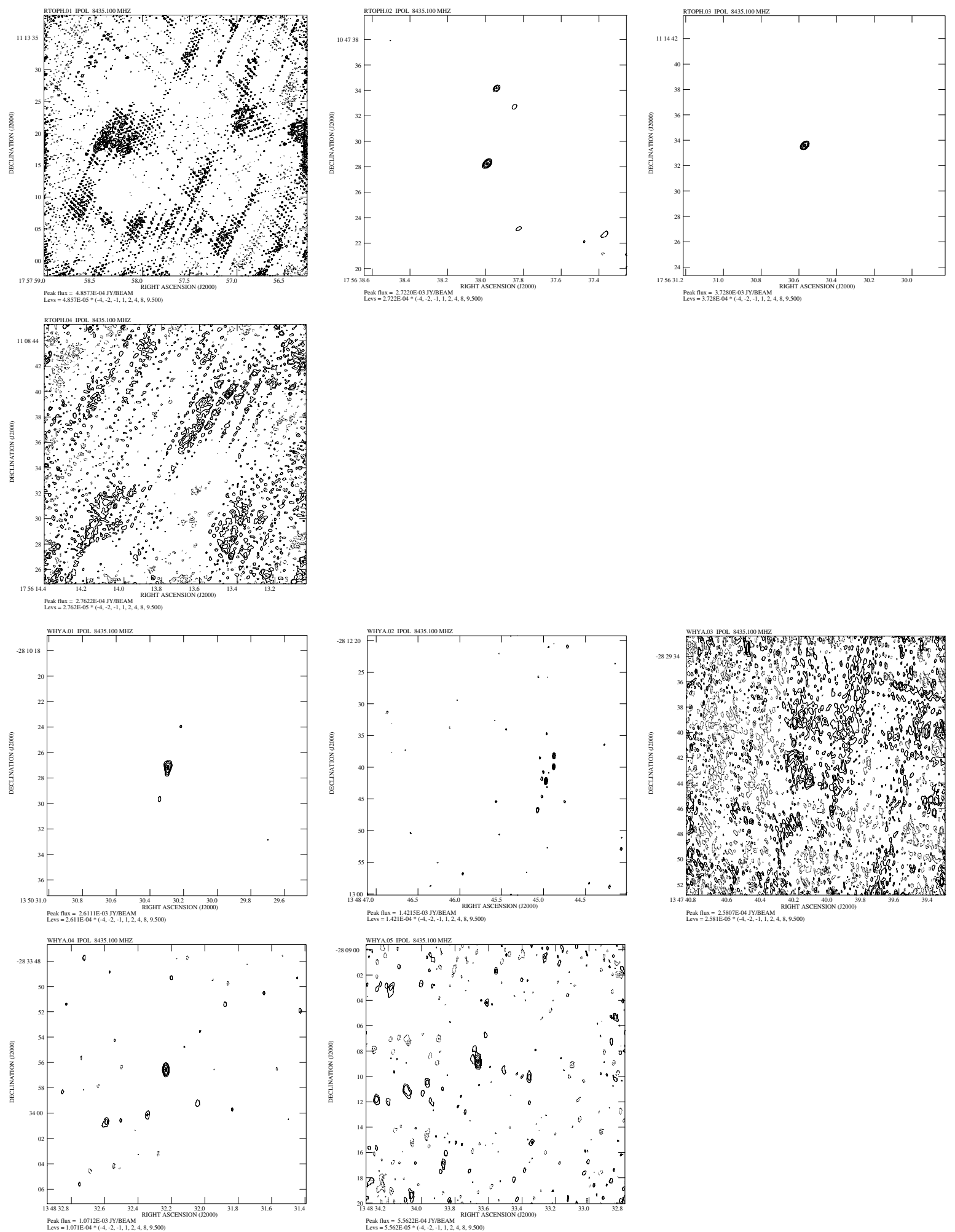

Fig. 9. As Fig. 6 for RT Oph and W Hya. None of the sources were of sufficient quality to be used as in-beam calibrators. 
W. H. T. Vlemmings and H. J. van Langevelde: Improved VLBI astrometry of OH maser stars, Online Material p 6

Table 3. X-Band VLA observation results. Results from the X-band VLA search for suitable in-beam calibrators. The sources were picked from the $1.4 \mathrm{GHz}$ NVSS survey (Condon et al. 1998). The observations were taken in D-configuration at May 21st 2003. The table gives the name identifying the nearby maser source with the NVSS name and integrated flux. Additionally, we give the coordinates of the peak emission, and the integrated and peak $8.4 \mathrm{GHz}$ flux determined using the AIPS task JMFIT, when a single component Gaussian fit was possible for the strongest component. The last column contains the seperation $\Delta \theta$ between the continuum source and the maser star in arcminutes. For those sources that were used as in-beam calibrators and detected in the VLBA $L$-band observations we also give JMFIT integrated fluxes.

\begin{tabular}{|c|c|c|c|c|c|c|c|}
\hline Source & NVSS name & $\begin{array}{c}\text { NVSS flux } \\
\text { (mJy) }\end{array}$ & $\begin{array}{c}\alpha_{\mathrm{J} 2000} \\
\text { hh mm ss } \\
\end{array}$ & $\begin{array}{c}\delta_{\text {J2000 }} \\
0,11\end{array}$ & $\begin{array}{c}\text { Flux } \\
(\mathrm{mJy}) \\
\end{array}$ & $\begin{array}{c}\text { Peak flux } \\
\text { (mJy/beam) }\end{array}$ & $\begin{array}{c}\Delta \theta \\
(\operatorname{arcmin})\end{array}$ \\
\hline RRAQL.01 & NVSS J195826-020352 & $116.8 \pm 3.5$ & 195826.5826 & -020351.833 & $13.57 \pm 0.19$ & $12.77 \pm 0.11$ & 16.5 \\
\hline RRAQL.02 & NVSS J195759-014012 & $115.5 \pm 4.1$ & 195759.5262 & -014014.670 & $21.00 \pm 0.08$ & $20.48 \pm 0.05$ & 14.2 \\
\hline RRAQL. $03^{a}$ & $\begin{array}{l}\text { NVSS J195655-013615 } \\
L \text {-band VLBI }\end{array}$ & $\begin{array}{l}94.3 \pm 2.9 \\
48.1 \pm 0.2\end{array}$ & 195655.2046 & -013615.426 & $70.96 \pm 0.12$ & $69.95 \pm 0.07$ & 19.8 \\
\hline RRAQL.04 & NVSS J195728-021337 & $36.6 \pm 1.2$ & & & & & 20.5 \\
\hline RRAQL.05 & NVSS J195627-014425 & $16.0 \pm 0.6$ & 195627.0759 & -014427.040 & $7.20 \pm 0.04$ & $7.14 \pm 0.02$ & 19.3 \\
\hline SCRB.01 & NVSS J152101+311537 & $175.5 \pm 5.3$ & 152101.2872 & +311537.796 & $97.48 \pm 0.69$ & $96.99 \pm 0.40$ & 8.1 \\
\hline SCRB. $02^{a, b}$ & $\begin{array}{l}\text { NVSS J152209+314414 } \\
L \text {-band VLBI }\end{array}$ & $\begin{array}{l}347.2 \pm 10.4 \\
252.7 \pm 0.1\end{array}$ & 152209.9937 & +314414.361 & $465.18 \pm 2.95$ & $458.56 \pm 1.69$ & 24.2 \\
\hline SCRB.03 & NVSS J152233+310911 & $51.3 \pm 1.9$ & & & & & 19.7 \\
\hline SCRB.04 & NVSS J152109+310334 & $21.3 \pm 0.7$ & 152109.7814 & +310334.369 & $2.44 \pm 0.11$ & $1.62 \pm 0.05$ & 18.7 \\
\hline SCRB.05 & NVSS J152012+310738 & $11.9 \pm 0.5$ & & & & & 20.9 \\
\hline SCRB.06 & NVSS J152111+312816 & $11.8 \pm 0.5$ & 152012.7797 & +310737.135 & $1.27 \pm 0.03$ & $1.11 \pm 0.01$ & 6.8 \\
\hline UHER.01 & NVSS J162605+191624 & $114.0 \pm 3.4$ & 162605.5769 & +191624.999 & $9.24 \pm 0.07$ & $2.63 \pm 0.02$ & 23.2 \\
\hline UHER. $02^{a}$ & $\begin{array}{l}\text { NVSS J162539+183727 } \\
L \text {-band VLBI }\end{array}$ & $\begin{array}{l}32.8 \pm 1.1 \\
4.5 \pm 0.3\end{array}$ & 162538.9655 & +183725.691 & $5.10 \pm 0.02$ & $3.92 \pm 0.01$ & 16.2 \\
\hline UHER.03 & NVSS J162435+185344 & $29.9 \pm 1.0$ & 162435.6826 & +185345.245 & $0.78 \pm 0.10$ & $0.29 \pm 0.03$ & 17.0 \\
\hline UHER.04 & NVSS J162544+184406 & $20.2 \pm 0.7$ & & & & & 9.5 \\
\hline UHER.05 & NVSS J162508+191647 & $20.0 \pm 1.1$ & & & & & 25.0 \\
\hline UHER.06 & NVSS J162508+190212 & $7.8 \pm 0.5$ & & & & & 12.7 \\
\hline UHER.07 & NVSS J162650+191246 & $12.8 \pm 0.6$ & 162650.0838 & +162650.0838 & $0.94 \pm 0.06$ & $0.37 \pm 0.02$ & 24.3 \\
\hline RAQL.01 $^{a}$ & $\begin{array}{l}\text { NVSS J190715+075240 } \\
L \text {-band VLBI }\end{array}$ & $\begin{array}{c}268.1 \pm 9.5 \\
1.1 \pm 0.2\end{array}$ & 190715.5506 & +075243.409 & $35.07 \pm 0.28$ & $20.10 \pm 0.11$ & 24.9 \\
\hline RAQL.02 & NVSS J190507+082958 & $200.0 \pm 6.0$ & 190507.0343 & +082957.507 & $8.95 \pm 0.07$ & $7.66 \pm 0.04$ & 24.6 \\
\hline RAQL.03 & NVSS J190552+075754 & $105.6 \pm 3.2$ & 190552.4491 & +075753.358 & $6.88 \pm 0.05$ & $3.80 \pm 0.02$ & 17.5 \\
\hline RAQL.04 & NVSS J190502+080239 & $56.0 \pm 3.5$ & & & & & 22.7 \\
\hline RCAS.01 & NVSS J235918+513815 & $58.9 \pm 1.8$ & 235919.0708 & +513815.198 & $9.97 \pm 0.07$ & $8.11 \pm 0.03$ & 17.1 \\
\hline RCAS.02 & NVSS J235959+511727 & $8.7 \pm 0.5$ & & & & & 15.9 \\
\hline RCAS.03 & NVSS J235908+510703 & $7.3 \pm 0.5$ & 235909.8053 & +510708.929 & $0.50 \pm 0.04$ & $0.47 \pm 0.02$ & 17.7 \\
\hline RCAS.04 ${ }^{a}$ & NVSS J000019+511254 & $6.9 \pm 0.5$ & 000019.3222 & +511254.106 & $17.49 \pm 0.17$ & $17.60 \pm 0.10$ & 20.7 \\
\hline RCAS.05 & NVSS J235735+510122 & $6.8 \pm 0.5$ & 235734.8546 & +510120.852 & $10.03 \pm 0.06$ & $10.06 \pm 0.03$ & 23.3 \\
\hline${\text { YCAS. } 01^{a}}^{a}$ & $\begin{array}{l}\text { NVSS J000108+552638 } \\
L \text {-band VLBI }\end{array}$ & $\begin{array}{c}289.7 \pm 8.7 \\
68.5 \pm 0.3\end{array}$ & 000108.7229 & +552638.668 & $33.93 \pm 0.11$ & $31.76 \pm 0.06$ & 23.5 \\
\hline YCAS.02 & NVSS J000156+554730 & $115.8 \pm 4.2$ & 000156.5666 & +554726.041 & $24.82 \pm 0.10$ & $24.56 \pm 0.06$ & 13.7 \\
\hline YCAS.03 & NVSS J000344+555625 & $84.4 \pm 2.6$ & 000344.1589 & +55 5626.483 & $7.80 \pm 0.07$ & $2.68 \pm 0.02$ & 15.9 \\
\hline YCAS.04 & NVSS J000339+552421 & $42.6 \pm 1.7$ & & & & & 16.7 \\
\hline RTOPH.01 & NVSS J175757+111318 & $58.8 \pm 2.1$ & & & & & 21.1 \\
\hline RTOPH.02 & NVSS J175637+104729 & $37.7 \pm 1.2$ & 175637.9921 & +104728.239 & $3.15 \pm 0.05$ & $2.70 \pm 0.03$ & 22.7 \\
\hline RTOPH.03 & NVSS J175630+111433 & $22.8 \pm 0.8$ & 175630.5689 & +11 1433.598 & $3.77 \pm 0.03$ & $3.70 \pm 0.02$ & 4.4 \\
\hline RTOPH.04 & NVSS J175613+110835 & $16.1 \pm 0.6$ & & & & & 4.9 \\
\hline WHYA.01 & NVSS J135030-281027 & $16.3 \pm 0.7$ & 135030.2936 & -281027.173 & $2.97 \pm 0.09$ & $2.56 \pm 0.05$ & 22.6 \\
\hline WHYA.02 & NVSS J134845-281239 & $9.9 \pm 0.6$ & 134844.9729 & -281242.180 & $1.49 \pm 0.05$ & $1.41 \pm 0.03$ & 10.1 \\
\hline WHYA.03 & NVSS J134740-282942 & $8.4 \pm 0.5$ & & & & & 19.6 \\
\hline WHYA.04 & NVSS J134832-283356 & $7.7 \pm 0.5$ & 134832.2336 & -283356.579 & $1.08 \pm 0.03$ & $1.08 \pm 0.02$ & 13.6 \\
\hline WHYA.05 & NVSS J134833-280909 & $7.3 \pm 0.5$ & 134833.6641 & -280908.811 & $0.67 \pm 0.04$ & $0.54 \pm 0.02$ & 14.3 \\
\hline
\end{tabular}

${ }^{a}$ In-beam calibrator used during VLBA observations.

${ }^{b}$ Also known as JVAS source J1522+3144 with previously know VLBI position. 
W. H. T. Vlemmings and H. J. van Langevelde: Improved VLBI astrometry of OH maser stars, Online Material p 7
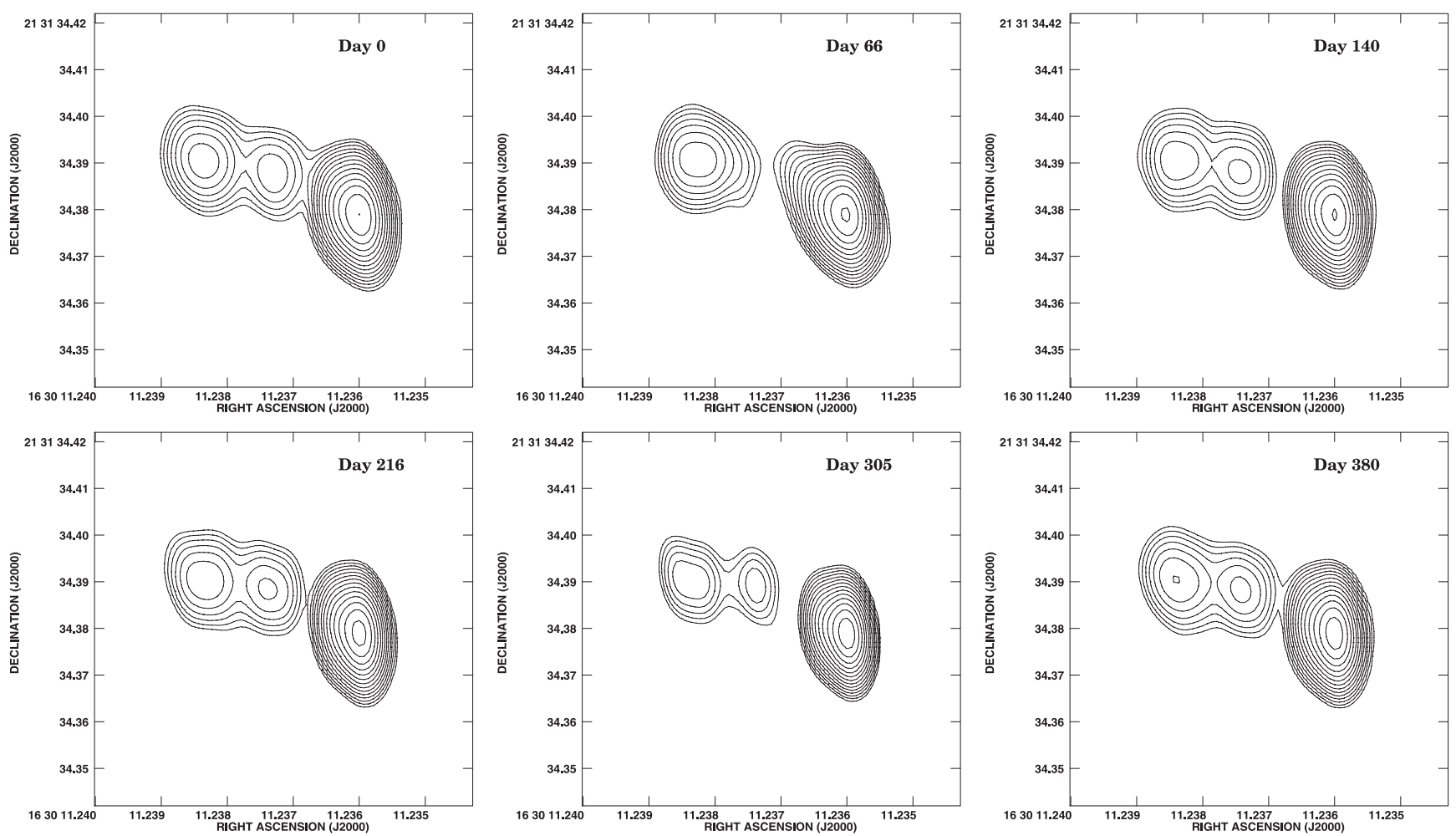

Fig. 10. The primary phase reference calibrator $\mathrm{J} 1630+2131$ used for U Her. The panels are labelled by the number of days after the first epoch of observations and include epoch $e$, for which we were unable to properly image the $\mathrm{OH}$ masers. The contours start at $1 \mathrm{mJy}$ and each subsequent contour level is multiplied by a factor of $\sqrt{2}$. 